HUTP-99/A065

hep-th/9912001

\title{
AdS/CFT and gravity
}

\author{
Steven S. Gubser \\ Lyman Laboratory of Physics, Harvard University, Cambridge, MA 02138, USA
}

\begin{abstract}
The radiation-dominated $k=0$ FRW cosmology emerges as the induced metric on a codimension one hypersurface of constant extrinsic curvature in the five-dimensional AdS-Schwarzschild solution. That we should get FRW cosmology in this way is an expected result from AdS/CFT in light of recent comments regarding the coupling of gravity to "boundary" conformal field theories. I remark on how this calculation bears on the understanding of Randall and Sundrum's "alternative to compactification." A generalization of the AdS/CFT prescription for computing Green's functions is suggested, and it is shown how gravity emerges from it with a strength $G_{4}=2 G_{5} / L$. Some upper bounds are set on the radius of curvature $L$ of $A d S_{5}$. One of them comes from estimating the rate of leakage of visible sector energy into the CFT. That rate is connected via a unitarity relation to deviations from Newton's force law at short distances. The best bound on $L$ obtained in this paper comes from a match to the parameters of string theory. It is $L \lesssim 1 \mathrm{~nm}$ if the string scale is $1 \mathrm{GeV}$. Higher string scales imply a tighter bound on $L$.
\end{abstract}

November 1999 


\section{Introduction}

The AdS/CFT correspondence [1, 2, 3] (see [4] for a review) relates a quantum field theory in one dimension to a theory in one higher dimension that includes gravity. The primary example is $\mathcal{N}=4$ super-Yang-Mills theory, which is related to the dimensional reduction of type IIB string theory on $S^{5}$ to the five-dimensional non-compact geometry $A d S_{5}$ (anti de-Sitter space). The boundary of the Poincaré patch of $A d S_{5}$ is simply Minkowski space, except that the metric on the boundary is only specified up to a conformal transformation. This is OK because $\mathcal{N}=4$ super-Yang-Mills theory is conformal, even at the quantum level. The correspondence is usually studied in a strong coupling region for the gauge theory, where it is far from classical, but the dual gravity picture is classical in the sense that curvatures are small on the Planck and string scales.

In [5] it was proposed that slices of $A d S_{5}$ could serve as an alternative to compactification manifolds. It was shown that when the near-boundary region of $A d S_{5}$ is cut away and the bulk spacetime simply ends on a wall of constant extrinsic curvature (a horosphere of $A d S_{5}$ to be precise), there is a normalizable graviton mode which has zero mass in the four dimensions of the boundary. The metric of $A d S_{5}$ is

$$
d s_{5}^{2}=e^{2 r / L}\left(-d t^{2}+d \vec{x}^{2}\right)+d r^{2}
$$

where $\vec{x}$ is an ordinary 3 -vector. This metric is a solution to $R_{\mu \nu}=-\frac{4}{L^{2}} g_{\mu \nu}$. Hypersurfaces of constant $r$ are horospheres. The part of the metric that is cut away in [5] is $r>r_{*}$ for some given $r_{*}$.

In fact the proposal of [5] was to glue two identical copies of the sliced anti-de Sitter space together along the $3+1$ dimensional boundary. However the four-dimensional graviton is quite a general phenomenon, and there is a large freedom in what one might have on the other side of the horospherical boundary of a given copy of $A d S_{5}$. An illustration of this can be found in [6], where a single copy of $A d S_{5}$ is obtained as part of a type IIB string compactification on an orientifold of $T^{6}$. The relevant point is that for a vacuum state of the theory, the extrinsic curvature of the boundary should be proportional to the induced metric:

$$
\Theta_{i j}=-\frac{1}{L} g_{i j}^{\text {(induced) }}
$$

Regarding the boundary as a positive tension $3+1$-dimensional brane separating two sliced copies of $A d S_{5}$, this amounts to the statement that the stress energy of the brane should respect $3+1$-dimensional Poincaré invariance. The constant of proportionality, $1 / L$, is required so that there is a balance between the brane tension and the bulk cosmological constant. More generally, a codimension one boundary of a five-dimensional space with no excitations on it should have the same property that $\Theta_{i j}=-\frac{1}{L} g_{i j}^{\text {(induced) }}$. 
The authors of [5] termed their construction an "alternative to compactification," which seemed appropriate because one can travel an infinite spatial distance into the five-dimensional bulk. Efforts such as [6] to realize the construction in string theory, using coincident D3-branes to produce the $A d S_{5}$ background, represent strongcoupling extrapolations of perturbative string compactifications, where the massless four-dimensional graviton is the usual zero-mode of the spin 2 closed string state. In such a picture, the Kaluza-Klein gravitons of [5] are interpreted as the strong-coupling description of open string excitations on the D3-branes, in line with the extensive literature on absorption by D3-branes [7, 8, 9].

It was suggested by J. Maldacena [10] that this "alternative to compactification" should properly be viewed in light of the AdS/CFT correspondence as a coupling of gravity to whatever strongly coupled conformal theory the $A d S_{5}$ geometry is dual to. This view was also taken by $\mathrm{H}$. Verlinde in $[6,11]$. A convincing statement of the case was made by E. Witten [12] in response to [13, 14].

From now on I will restrict myself to a minimal scenario where a single copy of $A d S_{5}$ is cut off by an end-of-the-universe brane. Such objects are well known in type I' string theory [15] and in Horava-Witten theory [16], so there is no problem of principle in having a true end of the universe. However my comments basically apply to any compactification geometry which involves the near-horizon part of $A d S_{5}$.

The idea that the scenario of [5] is best viewed in the context of AdS/CFT has not been universally embraced, perhaps partly because it is hard to see what to do with it. (That difficulty is not usually regarded as fatal, but it does seem to have held up progress on the current issue). The goal of section 2 is to make the idea seem more definite by using it to derive the radiation-dominated Friedmann-RobertsonWalker (FRW) cosmology. The approach is to change the bulk spacetime from $A d S_{5}$ to AdS-Schwarzschild, but not to excite any matter on the cutoff brane. The Hawking temperature of the AdS-Schwarzschild geometry, measured with respect to time on the cutoff brane, can be interpreted as the temperature of the CFT which the bulk spacetime is dual to. Readers determined to understand the construction from a braneworld perspective may find it most useful to think of the bulk as the background of near-extremal D3-branes. However, AdS/CFT allows us to reinterpret the entire AdS-Schwarzschild geometry as a manifestation of the dynamics of a four-dimensional conformal field theory at finite temperature. With the CFT interpretation in mind, it is easier to understand why the radiation-dominated FRW geometry emerges: all CFT's have the same equation of state up to numerical factors, so the FRW equations take the same form as they do in the radiation-dominated era of our universe.

The literature on brane-world cosmology is large and somewhat scattered; indeed I became aware of some recent overlapping papers only after this work was completed. Early work on supergravity domain walls in $A d S_{4}$ has been reviewed in [17]. Cosmo- 
logical solutions to Horava-Witten theory compactified on a Calabi-Yau manifold were investigated in [18]; see also [19] for subsequent development, and [20] for an analogous treatment of type I'. The works $[21,22,23,24]$ include constructions equivalent to equations (8)-(11), although the CFT interpretation was not offered. Reference [25] includes some formal developments and presents equations equivalent to (16). Discussions of cosmological constraints in $[23,26]$ have some overlap with section 4 . And there are a number of papers $[27,28,29,30,31]$ that use similar brane-world techniques. A more extensive list of references on brane-world cosmology can be found in [30]. The whole approach is rather different from the older string cosmology literature; see [32] for references.

Section 3 consists of some remarks on the general framework of AdS/CFT with a cutoff brane. A generalization of the prescription $[2,3]$ for computing Green's functions is suggested at the level of effective field theory. The four-dimensional Einstein action can be derived in this formalism, with the result $G_{4}=2 G_{5} / L$. This relation obtains regardless of the location of the cutoff brane in $A d S_{5}$. In standard AdS/CFT, where there is no cutoff brane and hence no normalizable graviton, the terms responsible for the Einstein action were removed using local counter-terms [33]. Corrections to Newton's force law are discussed from the CFT perspective, but I avoid presenting details since the idea of the calculation is not original to me.

Matter on the cutoff brane is incorporated naturally in the formalism. Although I do not propose a definite model, the idea is to have visible sector matter on the cutoff brane, somewhat as in certain heterotic M-theory models [34]. Excitations of that matter would have to dominate over the solution in section 2 at least for $z \lesssim 10^{10}$ in order for the cosmology to be realistic.

In section 4, I make some rough numerical estimates. One is to check the cosmological effects of visible sector matter losing its energy to the CFT. In AdS language this corresponds to absorption of bulk gravitons by the horizon of the Poincaré patch, as in $[7,8,9]$. The rate of energy loss is directly related to the deviations from Newton's force law. Another is to estimate parameters of string theory that would permit the deviations from Newton's force law to be observed experimentally, assuming that $A d S_{5}$ emerges from a string theory construction. To obtain deviations at the scale of even a nanometer (still three orders of magnitude below the sensitivity of proposed experiments) an extremely low string scale is required - approximately $1 \mathrm{GeV}$. I make some speculative remarks regarding low string scales at the end of section 4 .

Throughout the paper, $\mu$ will denote a five-dimensional bulk spacetime index and $i$ is a four-dimensional index. In cases where precision is required, I will denote fivedimensional coordinates as $x^{\mu}=(t, \vec{x}, r)$ and four-dimensional coordinates as $\xi^{i}=$ $(\tau, \vec{\xi})$. Throughout the paper, $\ell_{\mathrm{Pl}}$ will denote the four-dimensional Planck length: $\ell_{\mathrm{Pl}}=\sqrt{G_{4}} \approx 1.6 \times 10^{-33} \mathrm{~cm}$ in units where $\hbar=c=1$. 


\section{A cosmological solution}

Let us start purely from a four-dimensional point of view, and turn on a finite temperature for the conformal field theory which is small in Planck units. If we calculate the corresponding energy density $\rho$, use the trivial equation of state $p=\rho / 3$, and apply the standard equation

$$
\left(\frac{\dot{a}}{a}\right)^{2}=\frac{8 \pi G_{4}}{3} \rho
$$

what must result is the standard radiation-dominated FRW cosmology,

$$
d s^{2}=-d \tau^{2}+a(\tau)^{2} d \vec{x}^{2}
$$

where $a(\tau)^{2}$ is linear in $\tau .{ }^{1}$ I will always use $\tau$ for four-dimensional cosmological time; $t$ will be reserved for the Poincaré time in $A d S_{5}$. The only difficulty is finding the constant of proportionality in $\rho \propto T^{4}$ : if the conformal field theory is interacting, it could be a non-trivial exercise even in the limit $T \ll 1 / \ell_{\mathrm{Pl}}$ where gravity loops shouldn't matter. However if the theory in question has an AdS dual where the supergravity approximation is good, then the study of black holes in AdS guarantees the relation

$$
\rho=\frac{3 \pi^{2}}{2} c T^{4}
$$

where $c$ is the coefficient for the trace anomaly in a normalization where $c=\left(N^{2}-1\right) / 4$ for $\mathcal{N}=4 S U(N)$ super-Yang-Mills theory. ${ }^{2}$ In this normalization, a single Abelian photon has $c=1 / 10$. There is a standard relation in AdS/CFT [33]

$$
c=\frac{\pi}{8} \frac{L^{3}}{G_{5}} .
$$

It should be remarked that for $\mathcal{N}=4$ gauge theory at weak coupling, (5) becomes $\rho=2 \pi^{2} c T^{4}$. This is the $4 / 3$ problem, first noted in [36, 37], and now understood as being a result of strong interactions.

So far we have employed the AdS/CFT correspondence merely as a tool for determining a detail of the strong-coupling thermodynamics. However the calculation can be done entirely on the AdS side if we take seriously the idea that the cutoff brane is no more nor less than a coupling of gravity to the conformal field theory. It seems inevitable from a string theory perspective that the gravity would be quantized, and that the detailed "structure" of the cutoff brane encodes the details of the quantum gravity;

\footnotetext{
${ }^{1}$ There is probably no obstacle in principle to extending the calculation to positive spatial curvature. However for negative spatial curvature the pathology observed in [35] might emerge.

${ }^{2}$ The calculation of [33] shows that in the limit where classical gravity is applicable to the AdS black holes there is in fact only one independent coefficient in the trace anomaly. This and (5) are non-trivial constraints on theories which can have AdS duals.
} 
but by taking $T \ll 1 / \ell_{\mathrm{Pl}}$ we should be able to ignore this issue. The cutoff brane, or "Planck brane," controls gravity, while the bulk of $A d S_{5}$ controls the conformal field theory. By assumption, the Planck brane is not appreciably influenced by finite temperature, but the conformal field theory is; so we should retain (2), but change the bulk background from $A d S_{5}$ to AdS-Schwarzschild. The metric of AdS-Schwarzschild is

$$
\begin{aligned}
d s_{5}^{2} & =e^{2 r / L}\left(-h(r) d t^{2}+d \vec{x}^{2}\right)+\frac{d r^{2}}{h(r)} \\
h(r) & =1-b^{4} e^{-4 r / L} \\
b & =\pi L T_{0} .
\end{aligned}
$$

Here $T_{0}$ is the Hawking temperature associated with the time coordinate $t$. It is a constant parameter of the AdS-Schwarzschild solution. The calculation will deal only with the coordinate patch covered by $(t, \vec{x}, r)$.

Given an orientable surface with unit normal $n_{\mu}$ (which specifies a notion of outside and inside by the direction in which it points), the extrinsic curvature can be defined as $\Theta_{\mu \nu}=-\left(\delta_{\mu}^{\lambda}-n_{\mu} n^{\lambda}\right) \nabla_{\lambda} n_{\nu}$. We will follow [38] in taking $n_{\mu}$ to be the outward unit normal, which points toward the true boundary of $A d S_{5}$. There is a set of solutions to (2) which form a foliation of the coordinate patch in question:

$$
\frac{t}{L}=\frac{e^{r / L}}{b^{2}}+\frac{1}{4 b} \sum_{k=1}^{4} i^{k} \log \left(1-i^{k} b e^{-r / L}\right)+\frac{t_{0}}{L},
$$

where $t_{0}$ is a constant of integration specifying a particular leaf. All leaves have the same induced metric since they are related by translation in $t$. Using (2) with the same constant of proportionality, $1 / L$, avoids a four-dimensional cosmological constantmore about this later. It proves most convenient to parametrize a particular leaf by $\left(r_{*}, \vec{x}\right)$, where $r=r_{*}$ is a solution of (8) for $r$ in terms of $t$. Then the induced metric is

$$
d s_{\text {(induced) }}^{2}=-\frac{e^{4 r_{*} / L}}{b^{4}} d r_{*}^{2}+e^{2 r_{*} / L} d \vec{x}^{2}
$$

If we define

$$
\tau=L \frac{e^{2 r_{*} / L}}{2 b^{2}}
$$

then the metric (9) assumes the standard FRW form:

$$
\begin{aligned}
d s_{(\text {induced })}^{2} & =-d \tau^{2}+a(\tau)^{2} d \vec{x}^{2} \\
a(\tau) & =b \sqrt{\frac{2 \tau}{L}} .
\end{aligned}
$$

Thus we do indeed observe the linear $a(\tau)^{2}$ that we expected. This behavior is strictly a consequence of conformal invariance: any conformal field theory provides a source term for Einstein's equations just like a bunch of massless photons. 
We can be a little more quantitative and rederive the coefficient in (5) from the new perspective. In the late time limit, we can use the relation

$$
\frac{1}{G_{4}}=\frac{1}{G_{5}} \int_{-\infty}^{r_{*}} d r e^{2\left(r-r_{*}\right) / L}=\frac{L}{2 G_{5}} .
$$

Actually this relation comes from a Kaluza-Klein reduction of five-dimensional gravity to four for a horospherical Planck brane in pure $A d S_{5}$. It should be OK to leading order for a brane in an asymptotically $A d S_{5}$ region of bulk spacetime, provided the brane is only slightly curved on the scale $L$. Such a brane is locally like a horosphere of $A d S_{5}$. It is perhaps more common in the literature to see a modification of (12) that arises from removing the factor of $e^{-2 r_{*} / L}$ from inside the integral. The discrepancy is merely due to the fact that our four-dimensional metric is precisely the induced metric, whereas more commonly the four-dimensional metric on a horosphere is taken to be $e^{-2 r_{*} / L} d s_{\text {(induced) }}^{2}$. The form of (12) is forced on us by the choice $d s_{4}^{2}=d s_{\text {(induced) }}^{2}$, which does seem the natural one in the present context.

Combining the relation

$$
\frac{1}{4 \tau^{2}}=\left(\frac{\dot{a}}{a}\right)^{2}=\frac{8 \pi G_{4}}{3} \rho
$$

with (12) and (6) leads to

$$
\rho=\frac{3 b^{4}}{16 \pi G_{5} L e^{4 r_{*} / L}}=\frac{3 \pi^{2}}{2} c\left(e^{-r_{*} / L} T_{0}\right)^{4} .
$$

Now, the temperature $T_{0}$ measured with respect to the time $t$ is not the same as the temperature $T$ measured with respect to the time $\tau$; rather,

$$
T=\frac{d t}{d \tau} T_{0}=e^{-r_{*} / L} T_{0}
$$

where we have used the relation $t=\sqrt{2 L \tau} / b$. So (14) is indeed identical to (5), coefficient and all.

The foregoing calculation is more than a formal manipulation: it is an illustration that string theory on $A d S_{5}$ is identical to a 3+1-dimensional conformal field theory. We wanted our cosmology to be driven by the conformal field theory dual to the bulk AdS geometry rather than by anything on the Planck brane. So we left the Planck brane in its ground state and made the bulk AdS geometry thermal by adding a black hole horizon.

It is worth remarking that no stabilization mechanism was employed because none was needed. From a brane-world point of view, the worst has already happened: the negative tension brane of [39] has retreated to infinity, ${ }^{3}$ and the delicate near-horizon

\footnotetext{
${ }^{3}$ The reality of such a negative tension object is something I am only provisionally willing to allow for the sake of argument, since I am aware of no fully satisfactory string theory construction of it as yet in an AdS background.
} 
cusp has been cut off by a finite temperature horizon. The effect of that horizon is most transparent when viewed in light of the AdS/CFT correspondence: it means that the $3+1$-dimensional conformal field theory is at finite temperature.

In a generic bulk geometry, the retreating Planck brane would cause the fourdimensional Newton constant to change. In this regard, an asymptotically AdS space is very special: provided we use the induced metric on the Planck brane (rather than some warping of it) as the four-dimensional metric, (12) will hold asymptotically when the Planck brane is moving in the asymptotically AdS region with curvatures which are small compared to $L$.

That $d s_{\text {(induced) }}^{2}$ turned out to be exactly the radiation-dominated FRW metric should excite some suspicion. Mightn't there be quantum gravity effects at sufficiently early times which modify the picture? We derived the agreement between (5) and (14) using a late-time relation, (12). It seems like a massive conspiracy that the physics of early times would arrange for the cosmology to remain exactly radiation-dominated FRW. There are limits to what we can assert about physics at early times without specifying the nature of the Planck brane. It seems inevitable however that (2) will receive corrections at higher orders in derivatives.

Is this real cosmology? Not as it stands: nucleosynthesis would be dramatically spoiled if the "hidden CFT" that $A d S_{5}$ represents had any sizable effect on the radiationdominated era of our universe. However it is straightforward to extend the discussion by adding matter to the brane, and its stress tensor, $T_{i j}^{\text {(matter) }}$, could take over from $T_{i j}^{(\mathrm{CFT})}$ at late times. (If all we're worried about is nucleosynthesis, then late times means $z \lesssim 10^{10}$ ). The AdS/CFT equation relevant to such a scenario is

$$
\begin{aligned}
G_{i j}^{(\text {induced })} & -8 \pi G_{4} T_{i j}^{(\mathrm{CFT})}-8 \pi G_{4} T_{i j}^{(\text {matter })}= \\
- & \frac{2}{L}\left[\Theta_{i j}-\left(\Theta+\frac{3}{L}\right) g_{i j}^{(\text {induced })}\right]-8 \pi G_{4} T_{i j}^{(\text {matter })} .
\end{aligned}
$$

This equation is a rearrangement of a formula obtained in [38] in the course of deriving quasi-local stress-energy tensors for AdS/CFT in various dimensions. ${ }^{4}$ I have used (12) to define $G_{4}$. It is necessary to check that visible matter does not lose energy to the CFT fast enough to spoil the cosmology. Since the CFT's couplings are essentially of gravitational origin, this is perhaps plausible. An estimate will be presented in section 4 .

Actually, (16) is only an approximate statement of translation, via AdS/CFT, from purely four-dimensional quantities to quantities which constrain how the Planck brane sits in the five-dimensional spacetime. Equations with physical meaning arise from setting either side equal to zero. In this section we considered a case where $T_{i j}^{(\text {brane) }}=0$;

\footnotetext{
${ }^{4}$ Only I have changed the sign of the Einstein tensor. This is necessary because of a difference in sign conventions. The derivation in section 3 will serve as a check that the signs in (16) are consistent.
} 
then setting the right hand side equal to zero amounts to requiring (2). Solving (2) gave us back radiation-dominated FRW cosmology, which perhaps sounded surprising; but the identity (16) makes it inevitable, because if the right hand side vanishes, so must the left hand side.

Suppose now we knew all about the matter on the brane, and discovered that it generated a positive cosmological constant: $-8 \pi G_{4} T_{i j}^{\text {(matter) }}=\Lambda g_{i j}^{\text {(induced) }}$ with $\Lambda>0$. Assuming the AdS part to be at zero temperature, we would then reduce (16) to

$$
G_{i j}^{(\text {induced })}+\Lambda g_{i j}^{(\text {induced })}=-\frac{2}{L}\left[\Theta_{i j}-\left(\Theta+\frac{3}{L}+\frac{L^{2} \Lambda}{2}\right) g_{i j}^{(\text {induced })}\right] .
$$

It is straightforward to show that setting the right hand side equal to zero leads to a hypersurface in $A d S_{5}$ whose induced metric is $d S_{4}$. Approximately this calculation has appeared elsewhere in the literature, for instance [27, 28, 29, 31]. A direct analog in lower dimensions was treated in [17], where a fairly general discussion of induced metrics on codimension one domain walls in $A d S_{4}$ was also given.

\section{The general framework}

The equation (16) is an approximate first variation of a more general relation, which is the natural extension of the prescriptions of $[2,3]$ :

$$
\begin{aligned}
S_{\text {eff }}\left[\gamma_{i j}, \psi\right] & =S_{4 \text { gravity }}\left[\gamma_{i j}\right]+S_{4 \mathrm{~d} \text { matter }}\left[\gamma_{i j}, \psi\right]+W_{\mathrm{CFT}}\left[\gamma_{i j}\right] \\
& =\underset{g_{i j}^{\text {(induced) }}=\gamma_{i j}}{\operatorname{extremum}}\left(S_{\text {bulk }}\left[g_{\mu \nu}\right]+S_{\text {brane }}\left[g_{i j}^{\text {(induced) }}, \psi\right]\right) .
\end{aligned}
$$

The metric $\gamma_{i j}$ is the metric on the Planck brane, and $\psi$ are the extra matter fields which live on the Planck brane. The first equation indicates a natural way of splitting up the four-dimensional effective action into four-dimensional gravity, the CFT, and the four-dimensional matter which comes from excitations on the Planck brane. The second equation is the actual statement of AdS/CFT, which in this case includes a "brane reduction" of five-dimensional gravity to four dimensions, as envisaged in $[39,5]$. $W_{\mathrm{CFT}}$ is the generating functional of connected Green's functions of the conformal field theory, with a cutoff imposed at some energy scale $\Lambda$. See [40] for an early discussion of cutoffs in AdS/CFT.

One way to define the cutoff $\Lambda$ is as the energy of a fundamental string stretched from the Planck brane all the way to the horizon of $A d S_{5}$. This gives $\Lambda \sim L / \alpha^{\prime}$ if we measure energies with respect to a time $\tau$ on the Planck brane such that $\gamma_{\tau \tau}=-1$. There can however be ambiguities in normalizing $\Lambda$, depending on the physical question one is asking, as explained in [41]. If $A d S_{5}$ is generated as the background of many 
coincident D3-branes, then we can imagine peeling one of them off and bringing it close to the Planck brane. A fundamental string stretched from this D3-brane back to the main cluster has the interpretation of a massive $\mathrm{W}$-boson. In the supergravity description, this fundamental string stretches to the horizon of $A d S_{5}$. Thus the relation $\Lambda \sim L / \alpha^{\prime}$ has a simple motivation in terms of a BPS quantity, namely the mass for the heaviest W-boson which can be included in the effective theory by Higgsing the CFT. A comprehensive discussion of interactions might require a more precise specification of how a geometric cutoff in $A d S_{5}$ translates into a cutoff in the four-dimensional theory.

The extremum on the right hand side of (18) is taken subject to the boundary condition that the metric induced from $g_{\mu \nu}$ on the cutoff brane is $\gamma_{i j}$. It is the saddlepoint approximation to quantum gravity in the bulk. If we wanted to do quantum gravity in some more complete way (i.e. string theory), we would make the replacement

$$
\operatorname{extremum} S \rightarrow \frac{1}{i} \log \int[\mathcal{D} g] e^{i S}
$$

where $\int[\mathcal{D} g]$ represents path integration. (Path integration in the sense of (19) would amount to closed string field theory - a subject where our understanding is incomplete. We might however imagine some other way of improving the saddle point approximation). In a real string theory model, there would probably be many more bulk fields besides the metric $g_{\mu \nu}$ that $S_{\text {bulk }}$ would depend on, and they would also have to have their boundary values specified in the extremum (or path integral). $W_{\text {CFT }}$ would depend on those boundary values, and there would also be new terms added to $S_{4 \mathrm{~d} \text { gravity }}$ for the dynamics of the zero modes of the extra fields. How much of a problem all this extra junk is depends on the couplings to the standard model fields. The optimistic view is that such couplings are about as important in particle physics contexts as the coupling of electrons and quarks to gravity. The zero modes of extra bulk fields would modify long-distance four-dimensional gravity if they remained massless, but any sort of confinement or mass generation mechanism could prevent this problem.

Like all of AdS/CFT, (18) is a claim to be substantiated rather than an assumption. However, it is difficult to give a complete proof because $W_{\mathrm{CFT}}$ is a complicated nonlocal functional of $\gamma_{i j}$ whose exact form is independently accessible only through a strong coupling QFT computation. If one takes the boundary to be the true boundary of $A d S_{5}$, the evidence is compelling [4] that the extremum on the right hand side of (18) does indeed lead to the generating functional of connected Green's functions for a CFT. ${ }^{5}$ Through the UV-IR relation we understand that cutting off a portion of $A d S_{5}$ should change physics in the ultraviolet only. Thus (18) is true insofar as it is welldefined (that is, on the level of an effective field theory on energy scales much lower

\footnotetext{
${ }^{5} \mathrm{~A}$ conformal transformation on $\gamma_{i j}$ is needed as the cutoff is removed to keep $\gamma_{i j}$ finite. However, $W_{\mathrm{CFT}}$ without a cutoff only depends on the conformal class of $\gamma_{i j}$.
} 
than the cutoff $\Lambda$ ) provided we can show that $S_{4 \mathrm{~d} \text { gravity }}+S_{4 \mathrm{~d} \text { matter }}$ emerges from the extremum on the right hand side. That is what I will actually demonstrate concretely. In the process I will derive (12) in a general setting, and also check that the sign that seemed worrisome in (16) is OK.

The proof is piggy-backed on the calculations of [33]. In order to keep the presentation self-contained, I will recapitulate parts of that work. The setting is a foliation of a five-dimensional Einstein manifold $\mathcal{M}$ (for instance, $A d S_{5}$ or AdS-Schwarzschild), whose boundary has a metric in the conformal class of a specified metric $\bar{g}_{(0)}$, and whose metric can be written in the form [42]

$$
d s_{5}^{2}=g_{\mu \nu} d x^{\mu} d x^{\nu}=\frac{1}{4 \rho^{2}} d \rho^{2}+\frac{1}{\rho} \bar{g}_{i j} d \xi^{i} d \xi^{j} .
$$

In (20) and the following equations, $L$ has been set to 1 . The metric $\bar{g}_{i j}$ can depend on $\rho$, but according to $[33,42]$ it has an expansion

$$
\bar{g}=\bar{g}_{(0)}+\rho \bar{g}_{(2)}+\rho^{2} \log \rho \bar{h}_{(4)}+\rho^{2} \bar{g}_{(4)}+\ldots
$$

Here $\bar{g}_{(2)}$ and $\bar{h}_{(4)}$ are tensors constructed from $\bar{g}_{(0)}$ using two and four derivatives, respectively. The expansion breaks down after the logarithmic term, in the sense that the $\bar{g}_{(n)}$ are no longer covariant tensors. Fortunately the first two terms of (21) are all that we will need. Explicitly,

$$
\bar{g}_{i j}^{(2)}=\frac{1}{2}\left(\stackrel{o}{R}_{i j}-\frac{1}{6} \stackrel{o}{R} \bar{g}_{i j}^{(0)}\right)
$$

where $\stackrel{o}{R}_{i j}$ is the Ricci tensor of $\bar{g}_{i j}^{(0)}$ and $\stackrel{\circ}{R}$ is the associated Ricci scalar.

The action under the extremum in (18) is

$$
\begin{aligned}
& S_{\text {bulk }}\left[g_{\mu \nu}\right]+S_{\text {brane }}\left[g_{i j}^{\text {(induced) }}, \psi\right]= \\
& \quad \frac{1}{16 \pi G_{5}} \int_{\mathcal{M}} d^{5} x \sqrt{g}[R+20]+\frac{1}{16 \pi G_{5}} \int_{\partial \mathcal{M}_{\epsilon}} d^{4} \xi \sqrt{g^{\text {(induced) }}}[-2 \Theta+\alpha] .
\end{aligned}
$$

We have located the cutoff brane on the hypersurface $\partial \mathcal{M}_{\epsilon}$ defined by the equation $\rho=\epsilon$. We have also defined

$$
\alpha=\alpha_{0}+\frac{16 \pi G_{5}}{\sqrt{g^{\text {(induced) }}}} \mathcal{L}_{\text {matter }}\left(g_{i j}^{\text {(induced) }}, \psi\right) .
$$

The constant $\alpha_{0}$ is what we will adjust to balance the tension of the Planck brane against the bulk cosmological constant. An imperfect adjustment would lead to the $d S_{4}$ induced metric, as commented on after (17). Thus we are not claiming to make headway on the cosmological constant problem; rather, we are pushing it into the 
Planck brane. The extrinsic curvature term in (23) is necessary in order to have a well-defined variational principle.

Extremizing (23) subject to $g_{i j}^{\text {(induced) }}=\gamma_{i j}$ can be achieved by letting $d s_{5}^{2}$ have the form (20) and then setting $\bar{g}_{i j}=\epsilon \gamma_{i j}$ at $\rho=\epsilon$ (this is at least true up to errors which will be subleading in a derivative expansion). Then (cf. (10) of [33])

$$
\underset{g_{i j}^{\text {(induced) }}=\gamma_{i j}}{\operatorname{extremum}}\left(S_{\text {bulk }}\left[g_{\mu \nu}\right]+S_{\text {brane }}\left[g_{i j}^{(\text {induced) }}, \psi\right]\right)=\frac{1}{16 \pi G_{5}} \int d^{4} \xi \mathcal{L}
$$

where

$$
\begin{aligned}
\mathcal{L} & =4 \int_{\epsilon} \frac{d \rho}{\rho^{3}} \sqrt{|\operatorname{det} \bar{g}|}+\left[\frac{1}{\rho^{2}}\left(-8+4 \rho \partial_{\rho}+\alpha\right) \sqrt{\bar{g}}\right]_{\rho=\epsilon} \\
& =\sqrt{\left|\operatorname{det} \bar{g}_{(0)}\right|}\left[\frac{\alpha-6}{\epsilon^{2}}+\frac{\alpha}{2 \epsilon} \operatorname{tr} \bar{g}_{0}^{-1} \bar{g}_{2}-\log \epsilon \stackrel{o}{a}_{(4)}+(\text { finite })\right] .
\end{aligned}
$$

Here we have defined

$$
\stackrel{o}{a}_{(4)}=-\frac{1}{8} \stackrel{o}{R}^{i j} \stackrel{o}{R}_{i j}+\frac{1}{24} \stackrel{o}{R}^{2} .
$$

This quantity was identified in [33] as the conformal anomaly of the CFT. The AdS/CFT prescription as detailed there is simply to remove the terms that diverge as $\epsilon \rightarrow 0$ via local counterterms. This is the only sensible course if the ultimate goal is to take $\epsilon \rightarrow 0$ so that the cutoff boundary becomes the true boundary. Instead we want to keep the cutoff boundary at a finite, arbitrary $\epsilon$ and regard the induced metric $\gamma_{i j}$ on $\partial \mathcal{M}_{\epsilon}$ as the Einstein metric of the four-dimensional world. Rewriting (26) in terms of $\gamma_{i j}$, one finds

$$
\mathcal{L}=\sqrt{|\operatorname{det} \gamma|}\left[\alpha-6+\frac{1}{2} R-\log \epsilon a_{4}+\ldots\right]
$$

where now $R$ is the Ricci scalar of the metric $\gamma_{i j}$ and $a_{4}$ is defined as in (27), only using curvature tensors pertaining to $\gamma_{i j}$ rather than to $\bar{g}_{i j}^{(0)}$. One might fear that the logarithmic term in (21) would contribute to the $\log \epsilon$ term in (28). It doesn't because $\operatorname{tr} \bar{g}_{(0)}^{-1} \bar{h}_{(4)}=0$.

Because powers of $\epsilon$ cancel in (28) (and $\epsilon$ is finite anyway) there is no longer an expansion parameter in (28). The expansion can only be justified as a derivative expansion, provided that the embedding of the cutoff brane in the five-dimensional Einstein space involves only curvatures which are slight on the length scale $L$. Combining (24) and (25) with (28), setting $\alpha_{0}=6$, and repristinating powers of $L$, we find

$$
\begin{aligned}
& \underset{\substack{\text { (induced) } \\
\cos _{i j}}}{\operatorname{extremum}}\left(S_{\text {bulk }}\left[g_{\mu \nu}\right]+S_{\text {brane }}\left[g_{i j}^{\text {(induced) }}, \psi\right]\right)= \\
& \frac{L}{32 \pi G_{5}} \int d^{4} \xi \sqrt{\gamma} R+\int d^{4} \xi \sqrt{\gamma} \mathcal{L}_{\text {matter }}\left(\gamma_{i j}, \psi\right)+W_{\mathrm{CFT}}\left[\gamma_{i j}\right]
\end{aligned}
$$

where $W_{\mathrm{CFT}}$ includes the $\log \epsilon$ term in (28) plus all the other terms which we indicated with .... We indeed verify the relation $G_{4}=2 G_{5} / L$. Also, since the Ricci scalar came 


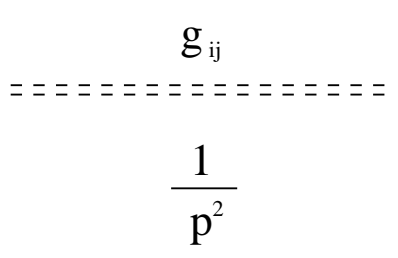

a)

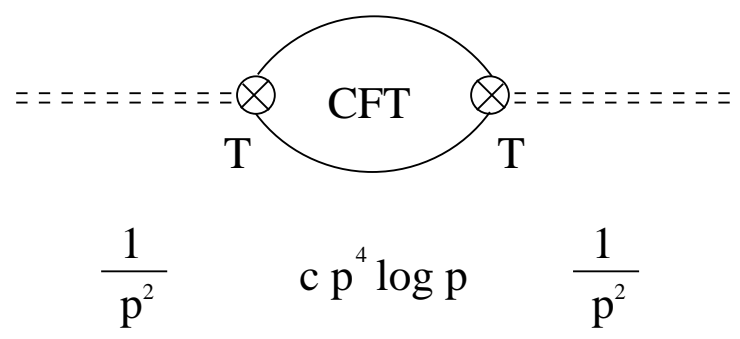

b)

Figure 1: Contributions to the graviton propagator, following [12]: a) free graviton propagation; b) leading CFT correction. The blob between the stress tensor insertions is intended to denote the full $\langle T T\rangle_{\mathrm{CFT}}$ correlator.

in with the right sign in (29), the signs of (16) are consistent. The calculation leading to (29) is similar to Kaluza-Klein reduction, the main difference being that the relation $G_{4}=2 G_{5} / L$ does not involve the total length of the fifth dimension (which could be infinite), but rather the curvature scale of the five-dimensional geometry. This makes the current scenario rather different from those of [43], where the circumference of the extra dimensions does affect the four-dimensional Planck length.

Extremizing (23) with respect to $g_{\mu \nu}$ without requiring $g_{i j}^{\text {(induced) }}=\gamma_{i j}$ would amount, at leading order in derivatives, to setting the right hand side of (16) to zero, as well as satisfying the bulk equations of motion. Given some information regarding the structure of the Planck brane, higher derivative corrections to (2) and to the right hand side of (16) would be accessible through a more meticulous treatment of this unrestricted extremization problem. The trick of (18) is to extremize first with the induced metric held fixed and then argue that the extremization that remains to be carried out gives us the equations of four-dimensional gravity (and brane matter if we want it), plus something non-local which we called $W_{\mathrm{CFT}}$. The claim that this something arises equivalently by integrating out a CFT below a cutoff $\Lambda$ is the substance of AdS/CFT and the basis for the suggestions in $[10,6,12]$.

The argument (20)-(29) stands in relation to the observation [5] of a normalizable graviton approximately as the derivation of the low-energy effective action of string theory via beta-functions stands in relation to the calculation of the massless string spectrum.

It should be possible to relate corrections to Einstein's equations and hence Newton's force law directly to the anomaly term $a_{4}$, proceeding along the lines of [44]. However it is more transparent to follow the analysis of [12], where we merely differentiate $W_{\mathrm{CFT}}\left[\gamma_{i j}\right]$ twice with respect to $\gamma_{i j}$ to obtain the first correction to the graviton propagator (see figure 1). The position space two-point function of the CFT stress tensor 
has the form $\langle T(x) T(0)\rangle \sim c / x^{8}$. In momentum space this is $\langle T(p) T(-p)\rangle \sim c p^{4} \log p$. The corrected graviton propagator is

$$
\begin{aligned}
G^{(2)}(p) & \sim \frac{1}{p^{2}}+\frac{1}{p^{2}} \ell_{\mathrm{Pl}}\left(c p^{4} \log p\right) \ell_{\mathrm{Pl}} \frac{1}{p^{2}} \\
G^{(2)}(x) & \sim \frac{1}{x^{2}}+\frac{c \ell_{\mathrm{Pl}}^{2}}{x^{4}}
\end{aligned}
$$

where the factors of the four-dimensional Planck length are vertex factors for the coupling of the stress tensor to the graviton. The altered propagator gives rise to deviations from Newton's $1 / r^{2}$ force law, estimated already from the AdS side in [5]:

$$
F=\frac{G m_{1} m_{2}}{r^{2}}\left(1+a_{1} \frac{L^{2}}{r^{2}}+\ldots\right),
$$

where $a_{1}$ is a dimensionless number on the order of unity. In (31) I have used (6) and (12) to combine $c \ell_{\mathrm{Pl}}^{2}$ into $\pi L^{2} / 4$. I do not claim any originality for the computation in (30) and (31). The only further addition I would make to the recorded comments in [12] is that the coefficient of leading correction is indeed computable from the CFT side: up to factors of order unity it is $G_{4}$ times the central charge of the CFT.

Clearly, by differentiating (18) and keeping track of all the Lorentz structure we could obtain the corrected propagator in complete detail and extract the exact value of $a_{1}$. I will refrain from entering into this computation here because another group is pursuing similar lines [45]. It was important however to present the general outline of the analysis because it will figure prominently in the next section.

\section{Bounds and estimates}

Note that (6) and (12) together imply that the central charge is $c=\frac{\pi}{4} \frac{L^{2}}{\ell_{\mathrm{Pl}}^{2}}$, where as usual $\ell_{\mathrm{Pl}}$ is the four-dimensional Planck length. For $A d S_{5}$ backgrounds arising from type IIB geometries including D3-branes, $c \sim N^{2}$ where $N$ is the number of D3-branes. So $N \sim L / \ell_{\mathrm{Pl}}$. To be definite, let us suppose that $L$ is on the order of a micron. Direct measurements of gravity already restrict $L \lesssim 1 \mathrm{~mm}$, and proposed experiments might probe Newton's force law to distances as small as a micron. $L \sim 1 \mu$ m means $N \sim 10^{29}$. This number seems on the high side for a string compactification: something has to soak up all the five-form flux. D3-brane charge is conserved, so it is true that if we managed to set $N=10^{29}$ through some arcane string theory construction, we wouldn't worry about it wiggling. As disciples of AdS/CFT we would also be relieved that fivedimensional quantum gravity effects aren't an immediate problem. However, a large hidden CFT is very dangerous in cosmology. Nucleosynthesis, for example, would be spoiled if $\rho_{\mathrm{CFT}} \gtrsim \rho_{\mathrm{SM}}$, where $\rho_{\mathrm{SM}}$ is the energy density of the Standard Model fields. 
Let us assume then that $\rho_{\mathrm{CFT}} \ll \rho_{\mathrm{SM}}$ around the time of nucleosynthesis. Because the CFT has a large number of degrees of freedom as compared to the Standard Model, this is possible only if the CFT is much colder than Standard Model excitations. Suppose that the Standard Model and the CFT are to a good approximation decoupled. Then $\rho_{\mathrm{CFT}}$ and $\rho_{\mathrm{SM}}$ decrease in fixed ratio during the radiation-dominated era, up to factors of order unity associated with freezing out the various massive fields of the Standard Model. In the matter-dominated era, $\rho_{\mathrm{CFT}}$ and $\rho_{C B R}$ decrease in fixed ratio. So we can guarantee that nucleosynthesis is unaffected by the CFT if we demand $\rho_{\mathrm{CFT}} \ll \rho_{\mathrm{CBR}}$ today. This translates roughly to $T_{\mathrm{CFT}} \lesssim T_{\mathrm{CBR}} / c^{1 / 4} \approx 10^{-14} \mathrm{~K}$ today if we want $L$ on the order of a micron. ${ }^{6}$ To summarize,

$$
c \sim N^{2}, \quad L \sim N \ell_{\mathrm{Pl}}, \quad T_{\mathrm{CFT}} \lesssim T_{\mathrm{CBR}} / \sqrt{N}
$$

Suppose the CFT is cold enough at some early time to satisfy $\rho_{\mathrm{CFT}} \ll \rho_{\mathrm{SM}} \cdot{ }^{7}$ Cosmology at later times could still be spoiled if energy leaks too quickly from visible matter into the CFT. The analogous problem in theories with compact extra dimensions is cooling by emission of bulk gravitons [46]. To evaluate whether there is a problem in our case, we must investigate the mechanisms of thermal equilibration between the CFT and the other matter in the universe, operating on the assumption that the CFT is very cold. Fortunately the tools are already partly in hand. Standard Model particles can lose energy to the conformal field theory through processes controlled by the graph in figure $2 \mathrm{a}$ ). The inclusive rates from these graphs are related to the $1 / r^{4}$ correction to Newton's law through the unitarity relation illustrated in figure $2 \mathrm{~b}$ ). In particular, the inclusive rate goes as $\ell_{\mathrm{Pl}}^{2} L^{2}$. By dimensional analysis the contribution they make to the loss of Standard Model energy density over time is

$$
\left(\frac{d \rho}{d \tau}\right)_{\text {lost }}=-a_{2} \ell_{\mathrm{Pl}}^{2} L^{2} T_{\mathrm{SM}}^{9},
$$

where $a_{2}$ is a dimensionless number of order unity and $T_{\mathrm{SM}}$ is the temperature of Standard Model excitations. From an AdS point of view, (33) is literally the rate at which energy density falls across the horizon to be absorbed by the D3-branes. Three powers of $T_{\mathrm{SM}}$ come from the absorption cross section $[7,8,9]$; also there are powers of $T_{\mathrm{SM}}$ from the finite temperature kinematics of the Standard Model particles. Energy

\footnotetext{
${ }^{6}$ We have used the AdS/CFT prediction $\rho \sim c T^{4}$. Naively counting flat directions in $\mathcal{N}=4$ super-Yang-Mills theory suggests $\rho \sim \sqrt{c} T^{4}$. Even if this were somehow true for a special CFT, it would only soften (32) to $T_{\mathrm{CFT}} \lesssim T_{\mathrm{CBR}} / N^{1 / 4}$.

${ }^{7}$ Section 2 treated the opposite limit. One should be able to use the equation (16) with $T_{i j}^{(\mathrm{CFT})}=0$ to find a hypersurface in $A d S_{5}$ whose induced metric is real-world cosmology. But this is only an equivalent means to find what we already know by solving Einstein's equations. In this section we will "cast down the ladder" and work directly in four dimensions whenever possible.
} 
a)

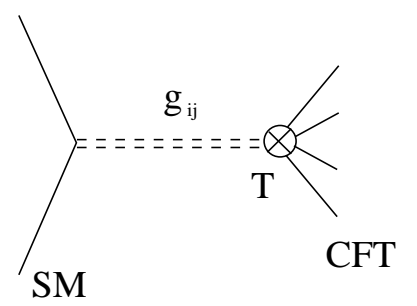

b)

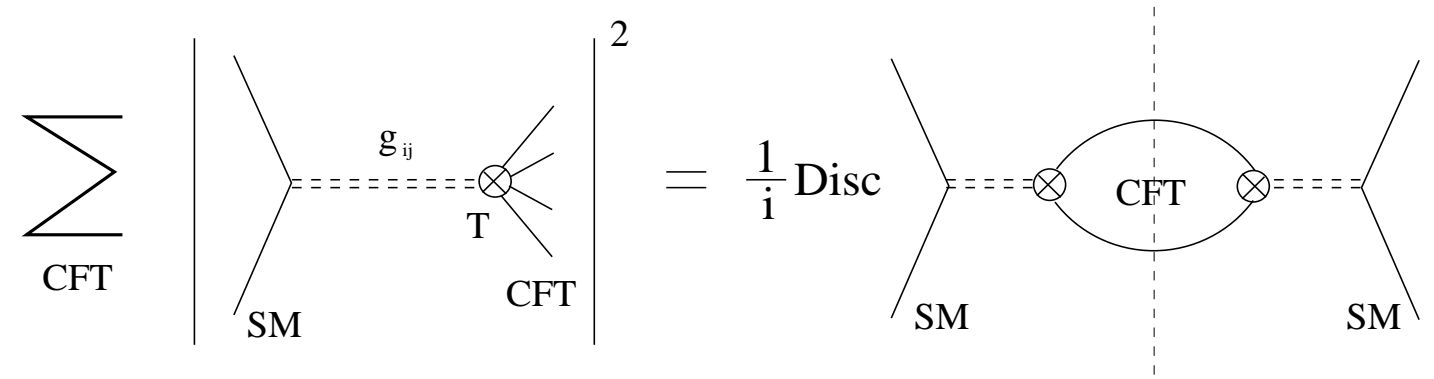

Figure 2: a) Standard Model particles losing energy via graviton exchange to CFT excitations. b) The inclusive rate is given by a unitarity cut of the first correction to the graviton propagator.

density also decreases because of Hubble expansion: in total,

$$
\frac{d \rho_{\mathrm{SM}}}{d \tau}=-\frac{\dot{a}}{a} T_{\mathrm{SM}}^{4}-\ell_{\mathrm{Pl}}^{2} L^{2} T_{\mathrm{SM}}^{9},
$$

where we have dropped factors of order unity. One such factor is the central charge of the Standard Model fields which are light compared to the temperature at any given time. Approximately this same factor appears in both terms on the right hand side of (34), so it doesn't matter much for the relative size of the terms. However $a_{2}$ does matter, and it should be computed if a more accurate estimate than the one presented here is desired. We have also suppressed a term in (34) for CFT energy leaking back into visible fields, but that is OK since we are operating on the assumption that the CFT is cold.

To determine whether the CFT is appreciably affecting the cosmology, one should compare the two terms in (34). Their ratio is

$$
\kappa=\ell_{\mathrm{Pl}}^{2} L^{2} T_{\mathrm{SM}}^{5} H^{-1}
$$

where $H^{-1}=a / \dot{a}$ is the inverse Hubble time (a function of $\tau$ ). The CFT will not appreciably affect cosmology as long as $\rho_{\mathrm{CFT}} \ll \rho_{\mathrm{SM}}$ and $\kappa$ is small. What small means in this context depends on all the "factors of order unity" that we have dropped. All these factors are calculable: once we have (6) and (12) the rest is essentially kinematics. 
In order to make some preliminary estimates I will assume that the Hubble expansion term in (34) dominates over the energy loss term when $\kappa \ll 1$.

The state of the universe today does not lead to a dramatic bound on $L$ : for instance, estimating $\kappa$ for the rate of energy loss from the CBR to the CFT gives

$$
L^{2} \ll \frac{1}{\ell_{\mathrm{Pl}} H_{o}^{-1} T_{\mathrm{CBR}}^{5}} \sim 10^{33} \mathrm{~cm}^{2}
$$

which is easily passed by any realistic theory. However the bound tightens as one goes back in time. Tracing the matter-dominated cosmology back to the time of last scatter at $z \sim 10^{4}$, one obtains roughly

$$
L^{2} \ll 10^{33} \mathrm{~cm}^{2}\left(\frac{a_{\text {last scatter }}}{a_{o}}\right)^{5} \frac{H_{o}^{-1}}{H_{\text {last scatter }}^{-1}}=10^{33} \mathrm{~cm}^{2}\left(\frac{a_{\text {last scatter }}}{a_{o}}\right)^{7 / 2}=10^{19} \mathrm{~cm}^{2},
$$

still not meaningfully restrictive. Tracing the radiation-dominated cosmology back to nucleosynthesis at $z \sim 10^{10}$, one obtains

$$
L^{2} \ll 10^{19} \mathrm{~cm}^{2}\left(\frac{a_{\text {nucleosynthesis }}}{a_{\text {last scatter }}}\right)^{5} \frac{H_{\text {last scatter }}^{-1}}{H_{\text {nucleosynthesis }}^{-1}}=10^{21} \mathrm{~cm}^{2}\left(\frac{a_{\text {nucleosynthesis }}}{a_{\text {last scatter }}}\right)^{3}=10 \mathrm{~cm}^{2} .
$$

Still this bound is satisfied with four orders of magnitude to spare (in $L$ ) if we suppose $L$ to be on the order of a micron. I emphasize the extreme simple-mindedness of the estimates: all I have done in (38) is to write

$$
L^{2}=\kappa \frac{1}{\ell_{\mathrm{Pl}}^{2} T_{\mathrm{SM}}^{5} H^{-1}} \approx \kappa \frac{1}{\ell_{\mathrm{Pl}}^{2} T_{\mathrm{CBR}}^{5} H_{o}^{-1}} \frac{1}{z_{\text {last scatter }}^{7 / 2}}\left(\frac{z_{\text {last scatter }}}{z_{\text {nucleosynthesis }}}\right)^{3},
$$

and then demand $\kappa \ll 1$. The powers of $z$ in (37)-(39) arise from the relations $H^{-1} \sim$ $a^{3 / 2}$ for the matter dominated cosmology and $H^{-1} \sim a^{2}$ for the radiation-dominated cosmology. In view of the actual number obtained in (38), a more accurate estimate would be desirable. One can also attempt to trace cosmology back to larger $z$ and tighten the bound on $L$ further, if one feels convinced that $\kappa$ must still be small for $z>10^{10}$.

An independent bound on $L$ could obtained by checking the effect on supernovas of energy loss to the CFT, as in [46]. The energy scales here are on the order of $30 \mathrm{MeV}$, so a slightly better bound than (38) might be expected.

There is yet another way to set a bound on $L$ if we assume that the $A d S_{5}$ geometry comes from type IIB string theory through some Freund-Rubin ansatz or related compactification. In such compactifications, the extra five dimensions have the same length scale $L$ as $A d S_{5}$. Suppose $A d S_{5} \times S^{5}$ is the relevant geometry. Then the standard string 
theory relation $16 \pi G_{10}=(2 \pi)^{7} g_{s}^{2} \alpha^{4}$ combined with (12) and $\operatorname{Vol} S^{5}=\pi^{3} L^{5}$ leads us to

$$
L=g_{s}^{1 / 3}\left(\frac{16 \pi^{3} \alpha^{\prime 4}}{G_{4}}\right)^{1 / 6} .
$$

Type IIB theory has an S-duality symmetry which takes $g_{s} \rightarrow 1 / g_{s}$. Thus we can assume that $g_{s} \leq 1$. A conventional value of $\sqrt{\alpha^{\prime}}$ would be only a few times the fourdimensional Planck length, $\ell_{\mathrm{Pl}}$. This results in a bound on $L$ which is also a few times $\ell_{\mathrm{Pl}}$. In order to make $L$ observably big, we would have to make $\sqrt{\alpha^{\prime}}$ big too. What is the biggest $\sqrt{\alpha^{\prime}}$ we could possibly imagine? In the old days of string theory the answer would have been $\sqrt{\alpha^{\prime}} \sim 1 \mathrm{GeV}^{-1} \approx 0.2 \mathrm{fm}$ : this is literally the Regge slope of observed hadronic spectra. In recent literature [47], values of $\sqrt{\alpha^{\prime}}$ as big as $1 \mathrm{TeV}^{-1}$ have been regarded as acceptable. Plugging these numbers into (40) leads to

$$
\begin{aligned}
L \lesssim 10^{-7} \mathrm{~cm} & \text { for } \sqrt{\alpha^{\prime}} & \sim 1 \mathrm{GeV}^{-1} \\
L \lesssim 10^{-11} \mathrm{~cm} & \text { for } \sqrt{\alpha^{\prime}} & \sim 1 \mathrm{TeV}^{-1} .
\end{aligned}
$$

The bad news is that deviations from Newton's force law on length scales this small won't be detected any time soon. The good news is that standard cosmology is no problem, as far back as nucleosynthesis and further. If we assume that the radiationdominated FRW solution still pertains, we can estimate the redshift $z_{*}$ and the thermal energies $T_{*}$ at which $\kappa=1$. The result is

$$
\begin{array}{rlrlrl}
z_{*} & \sim 10^{15}, & T_{*} & \sim 100 \mathrm{GeV} & & \text { for } \sqrt{\alpha^{\prime}} \sim 1 \mathrm{GeV}^{-1} \\
z_{*} \sim 10^{18}, & T_{*} \sim 100 \mathrm{TeV} & & \text { for } \sqrt{\alpha^{\prime}} \sim 1 \mathrm{TeV}^{-1} .
\end{array}
$$

To get the numbers in (42) we have combined several approximations and assumptions. The "error in the exponent" should probably be taken to be about \pm 2 . It is somewhat suggestive that the values of $T_{*}$ we found are "within errors" of the boundary of our direct knowledge of particle physics. If the string scale is at a $\mathrm{TeV}$, then physics changes sufficiently there that we can no longer have any confidence that the radiationdominated FRW cosmology is relevant. Thus the second line of (42) only shows that there are no cosmological problems as far back as we can trace the theory. Strings at a $\mathrm{GeV}$ are a different matter, and we will return to them shortly.

Although type IIB string theory provides the best-understood vacua involving $A d S_{5}$, it is conceivable that some other type of string theory, even a non-critical string, could have an $A d S_{5}$ vacuum: see for example [48]. For a non-critical string, (40) would not be the right estimate, since some or all of the five compact dimensions simply aren't there. Suppose the non-critical string lives in $n$ dimensions, with $n \geq 5$. Assume also that it exhibits some form of S-duality, so that the coupling cannot be parametrically large. Then

$$
L \lesssim\left(\frac{\sqrt{\alpha^{\prime}}}{\ell_{\mathrm{Pl}}}\right)^{\gamma} \sqrt{\alpha^{\prime}}
$$


where $\gamma=2 /(n-4)$. If we allow $n$ to range from 10 to 5 , the corresponding range of $\gamma$ is from $1 / 3$ to 2 . It is also conceivable that some intersecting configuration of branes in critical string theory could have an $A d S_{5}$ component in its near-horizon geometry, and a different relation from (40) could pertain if some of the branes had more than $3+1$ worldvolume dimensions. I am not currently aware of any completely well-defined, non-critical string theory other than the $c \leq 1$ toys. Nor can I give a string theoretic example of intersecting branes with an $A d S_{5}$ near-horizon geometry. Besides, if the extra dimensions of the intersecting branes are larger than $L$, then the salient physics of extra dimensions would be more along the lines of [43] than [5]. For the sake of a concrete discussion, let us stick to (40), with (43) as a possible alternative.

Once we have ventured to set $\sqrt{\alpha^{\prime}} \approx 1 \mathrm{GeV}^{-1}$, the burning question is why all collider physics from a GeV up to a TeV isn't dramatically different. The simplest answer is rather iconoclastic. It is that from a four-dimensional point of view, strings are nothing more than QCD flux tubes. For energies well above $1 \mathrm{GeV}$, but below the cutoff scale $\Lambda \sim L / \alpha^{\prime}$, a better set of variables is the particles of the Standard Model, plus a massless propagating graviton. In the low-energy regime where strings are the good variables, there is a massless graviton in the closed string spectrum. The graviton must be present in a description of the theory at any scale: on very general grounds [49] it is impossible for the graviton to be a composite particle. ${ }^{8}$ The spectrum could also include massless open strings if the Planck brane involves D-branes. The gluons in the Standard Model lagrangian might be represented in this way at low energies. Intuitively, the reason why a disk diagram with two gluon boundary insertions and one bulk insertion of a graviton wouldn't couple gluons to gravity on the scale of femtometers is that the wavefunction overlap is small. This is the magic of extra dimensions (exploited similarly in [43]): (40) is roughly a condition on how big the extra dimensions have to be in the well-understood type IIB string theory examples to make low-energy strings consistent with gravity at the four-dimensional Planck scale. The modified relation (43) could be pertinent for alternative models, as discussed in the previous paragraph.

The view taken in the previous paragraph is distinct from those of [50] or [6]. Wilson loops in AdS/CFT usually seek out a location of large redshift in the bulk geometry in order to lower their tension to the scale of confinement. The current scenario has Wilson loops terminating on the Planck brane, and the relevant geometry is the geometry near the Planck brane. I would not exclude scenarios where a large redshift does exist near the Planck brane, and the parameter entering into the Regge relation is a redshifted $\alpha^{\prime}$. If that is the way we think QCD strings are realized, then once again the bound on $L$ is tighter than $L \lesssim 1 \mathrm{~nm}$, since the $\alpha^{\prime}$ that enters (40) is the un-redshifted string tension.

\footnotetext{
${ }^{8}$ I thank M. Strassler and R. Sundrum for discussions on this point and related issues.
} 
Strings at a $\mathrm{GeV}$ seem like a natural apotheosis of the proposals of $[43,47]$. We do not have to "get rid" of the graviton if there are extra dimensions on the scale of a nanometer. (Significantly smaller $L$ would work in a model where (43) pertains). We do not have to worry about nucleosynthesis if the estimate (38) bears out. But we do have to face some hard questions. First, if $L \sim 1 \mathrm{~nm}$, how do we manage to accommodate $N=L / \ell_{\mathrm{Pl}} \approx 10^{26}$ D3-branes? Something has to soak up all the Ramond-Ramond flux, and that sounds like an impossible stretch for string compactifications (see for example [6]). $N$ comes out somewhat smaller if $g_{s}$ is small, or in models where (43) pertains with $n<10$. Second, string theory would have to face up to hadron physics in the energy range between pions and partons. Regge trajectories are as suggestive as they always were, but there is much more to be explained. Processes where some or all of the final energy winds up in CFT excitations are likely to be a problem. However the relevant branching ratios typically depend on $L$ rather than $\alpha^{\prime}$, and amount to yet another way of setting an upper bound on $L$. Third, strings could stretch from the Planck brane all the way into the $A d S_{5}$ bulk (to connect with a D3-brane if one wants to think in those terms) at only a finite cost in energy. The mass of such a string is roughly $L / \alpha^{\prime}$, which comes out to be approximately $3000 \mathrm{TeV}$ if we use $L \sim 1 \mathrm{~nm}$. This is out of the range of colliders, but it is nevertheless a dangerous number for any sort of loop computation because these strings are so numerous: there are as many of them as there are D3-branes. Their mass is bigger if (43) applies: $L / \alpha^{\prime} \sim 10^{18 \gamma} \mathrm{GeV}$. Fourth and finally, if flux tubes are long strings ending on the Planck brane, then what are quarks?

As observed in section 3, there is a precise way of characterizing the strings stretched from the Planck brane to the horizon of $A d S_{5}$ : they are the massive $W$ bosons associated with the separation of the Planck brane from the $L / \ell_{\mathrm{Pl}} \mathrm{D} 3$-branes that create the $A d S_{5}$ geometry. The scale of these masses could be lowered, say to $30 \mathrm{TeV}$, if $L$ falls sufficiently short of saturating the bound in the first line of (41). Or we could return to strings at a $\mathrm{TeV}$ and get approximately the same $30 \mathrm{TeV}$ Higgs scale by saturating the bound in the second line of (41). Either way, we are left with a version of [51], only with an enormous hidden sector gauge group and strings at a $\mathrm{GeV}$ or a $\mathrm{TeV}$. In [51], it seemed like coupling the CFT to gravity might resurrect the hierarchy problem. This is less of a problem if the string scale is smaller than or comparable to the scale of soft breakings of the CFT: one may hope that stringy "softness" ameliorates the divergences of gravity already at the string scale. There is no clear microscopic picture of what the theory is without specifying the nature of the Planck brane. However, the relation (40) between Newton's coupling and other low-energy quantities should not depend on the detailed properties of the Planck brane.

In conclusion, insisting that $A d S_{5}$ has to come from string theory provides a bound on $L$ which is sharper than we were able to obtain from nucleosynthesis, and which 
appears to rule out experimental observation of (31). There are two reasons why string theory demands a small $L$. First, $L / \ell_{\mathrm{Pl}} \sim N$, where $N$ is the number of units of Ramond-Ramond five-form flux. It is hard to make this number really big in string compactifications. Second, $G_{4} L^{6} \lesssim \alpha^{\prime 4}$, so we can only get big $L$ if we allow big $\alpha^{\prime}$. In an attempt to be maximally optimistic about the size of $L$, we have reconsidered strings at a $\mathrm{GeV}$. Even this radical step only gave us $L \lesssim 1 \mathrm{~nm}$. If we make $\alpha^{\prime}$ even bigger, it only heightens the difficulties we encountered trying to make sense of $\mathrm{GeV}$ strings. The strategies proposed in AdS/CFT contexts to relate strings to QCD flux tubes generally have the property that the fundamental $\alpha^{\prime}$ is smaller than $1 \mathrm{GeV}^{-2}$, implying a tighter bound on $L$.

\section{Discussion}

The FRW cosmology found in section 2 is an interesting check of the claim that the "alternative to compactification" proposed in [5] is equivalent to a cutoff conformal field theory coupled to four-dimensional gravity. However, as emphasized in section 4, the CFT shouldn't make any sizeable contribution to the actual cosmology of our universe at times later than $z=10^{10}$. Before that time, one is entitled to speculate about the physical relevance of the solution of section 2. Suppose that the CFT and the visible sector matter on the Planck brane were in thermal equilibrium at some early time. Assuming that the CFT has a much larger central charge, we have $\rho_{\mathrm{CFT}} \gg \rho_{\text {matter }}$, and the solution found in section 2 should approximately describe the cosmology. At late times one needs $\rho_{\text {CFT }} \ll \rho_{\text {matter. }}$. In parallel with [46], we might imagine an inflationary scenario where the inflaton lives on the Planck brane. Then reheating directly affects only the visible sector, and if $\kappa$ is small by the time of reheating there is substantially no thermal equilibration with the CFT.

In a scenario with $\mathrm{GeV}$ strings, thermalization with the CFT sets in significantly around $100 \mathrm{GeV}$ (although we must recall that the estimates here were extremely crude). That alone might lead us to rule this case out unless a reheating mechanism could be proposed at a lower scale.

In known string compactifications, the number $N$ of D3-branes is typically on the order of 10. As many as $10^{3}$ D3-branes were claimed to be attainable in certain orbifold examples [6]. If we take this as a strict bound, then the relation $L / \ell_{\mathrm{Pl}} \sim N$ puts our entire discussion at an inaccessibly small length scale: $L \sim 10^{-30} \mathrm{~cm}$ for $N=10^{3}$. (As usual, $\ell_{\mathrm{Pl}}$ is the four-dimensional Planck scale). The formalism developed in section 3 could still be useful for extracting a "low-energy" effective theory- "lowenergy" being interpreted now as much less than $10^{16} \mathrm{GeV}$. Standard inflation occurs around $10^{14} \mathrm{GeV}$, so it is possible one might embed a "conventional" inflationary model in $A d S_{5}$ using the $d S_{4}$ solution discussed after (17). The amusing aspect of such a 
model is that there is a natural candidate for the pre-inflationary universe: it is the radiation-dominated FRW solution found in section 2.

There are two solid conclusions to be drawn from the estimates of section 4. First, our present understanding of nucleosynthesis would not be threatened if deviations from Newton's force law of the form (31) were found. We already know that such deviations cannot be present on scales much larger than a millimeter, and this is enough to suppress the associated loss of energy to the conformal field theory for $z$ as large as $10^{10}$. Second, string theory as we understand it seems to forbid an $A d S_{5}$ space large enough to cause measurable deviations from Newton's force law. Even if we are willing to take the string scale down to $1 \mathrm{GeV}$ and regard strings as collective effects of QCD, $L$ still can't be larger than $1 \mathrm{~nm}$.

There is nothing sacred about an $A d S_{5}$ bulk spacetime: it has been the focus of so much recent literature in part because it is simple. Practically any string theory realization of $A d S_{5}$ will include scalar fields, and if they have a non-trivial profile, large deviations from $A d S_{5}$ are the generic behavior far from the boundary. The literature on renormalization group in AdS/CFT flows provides ample evidence of this (see for example $[52,53,54,55,56,57])$. Only a subset of these geometries can support finite temperature, due to boundary conditions on the scalars at the black hole horizon. A felicitous feature of $A d S_{5}$, which will not be shared by generic "RG flow" geometries, is that the relation $G_{4}=2 G_{5} / L$ obtains no matter where the Planck brane is in the bulk geometry. The formalism worked out in section 3 will still retain its general features in a more generic bulk geometry, but details will be rather different: for instance, it is no longer clear that the induced metric on the cutoff brane will be the Einstein frame metric.

A cutoff brane in a bulk geometry whose AdS/CFT dual is a quantum field theory undergoing renormalization group flow corresponds to gravity coupled to that same QFT. The proposal of [39] is to put the Standard Model not on the cutoff brane, but rather on some brane far from the boundary, where $g_{t t}$ is very small. There are several ways that such a construction could be realized in string theory. First, if the five-dimensional space-time ends at a finite minimum of $g_{t t}$, then one can show that the end-of-the-world brane must have negative tension. The best-understood constructions in the current literature which admit negative tension end-of-the-world branes are type I' string theory and certain Calabi-Yau compactifications of HoravaWitten theory. At the classical level, these constructions do not allow an $A d S_{5}$ bulk: there is always some scalar that evolves across the five-dimensional bulk. There is not yet compelling evidence that all scalars could be held fixed and an $A d S_{5}$ bulk obtained. It would be possible to develop a formalism similar to the one in section 3 for type $\mathrm{I}^{\prime}$ or Horava-Witten constructions, but it would have more the flavor of an ordinary KaluzaKlein reduction, where heavy fields are integrated out and light fields are kept. The 
distinctive feature of (18) is that it enables us to obtain a non-local functional which summarizes the dynamics of infrared degrees of freedom.

If there are no negative tension branes, the only option is for the five-dimensional space-time to continue all the way to $g_{t t}=0$. If there are scalars involved, the generic behavior is for curvatures to become strong as $g_{t t} \rightarrow 0$. AdS/CFT has limited computational power in such circumstances. The best hope is that string theory provides a resolution of the strong curvatures. If visible sector fields live on branes at strong curvatures, then we are not in a position to say much about the physics. It is also conceivable [58] that visible sector fields live on a probe brane at small but nonzero $g_{t t}$. There are potential phenomenological virtues to such a model, but it seems somewhat contrived.

String theory and string dualities have taught us that extra dimensions are theoretically inexpensive. But the view of the fifth dimension espoused in the current paper is not excessively literal: rather than making the claim that there is actually a large extra dimension of space waiting to be discovered, the statement is that an extra dimension is a convenient way to describe collective phenomena of a strongly coupled quantum field theory - in the present case, a conformal field theory coupled to gravity. To make this seem more definite, suppose measurements of gravity at a micron did after all turn up deviations from Newton's law of the form (31). The "AdS" interpretation would be that gravitons are propagating in the fifth dimension, while the "CFT" interpretation would be that a loop of gauge bosons in a purely four-dimensional theory had contributed. Which interpretation we prefer is a matter of ontology: if AdS/CFT is right then they are absolutely indistinguishable on experimental grounds. My current ontology isn't very happy either with a CFT with $c \sim 10^{58}$ or with a fifth dimension with curvatures on the scale of a micron. But it is in the subtle guises of string duality and string compactification that I suspect extra dimensions have the best chance of improving our understanding of the physical world.

\section{Acknowledgements}

Although it is tricky to assign credit in the absence of publications, I should acknowledge the important contributions of J. Maldacena and E. Witten. As far as I can ascertain, J. Maldacena was the first to enunciate the view that the Randall-Sundrum "alternative to compactification" is nothing more nor less than gravity coupled to a strongly interacting CFT; and as far as my personal knowledge extends, E. Witten was the first to suggest a definite calculation based on the idea, namely a correction to Newton's law based on the two-point function of the stress tensor of the CFT. I have also profited greatly from conversations with H. Verlinde. I thank the participants of the conference "New Dimensions in String theory and Field theory" at the ITP in Santa 
Barbara for lively discussions - especially I. Klebanov, M. Gremm, R. Myers, V. Periwal, B. Ovrut, K. Skenderis, S. Giddings, S. Elitzur, E. Witten, V. Hubeny, L. Bildsten, R. Maimon, R. Sundrum, M. Strassler, and G. Horowitz. I thank N. Warner, K. Pilch, D. Freedman, O. DeWolfe, A. Karch, E. Silverstein, and S. Kachru for earlier discussions, and particularly D. Gross for reading an early version of the manuscript and for useful comments.

This research was supported by the Harvard Society of Fellows, and also in part by the NSF under grant number PHY-98-02709, and by DOE grant DE-FGO2-91ER40654. I thank the ITP at Santa Barbara for hospitality while the work was carried out.

\section{References}

[1] J. Maldacena, "The Large N limit of superconformal field theories and supergravity," Adv. Theor. Math. Phys. 2 (1998) 231, hep-th/9711200.

[2] S. S. Gubser, I. R. Klebanov, and A. M. Polyakov, "Gauge theory correlators from noncritical string theory," Phys. Lett. B428 (1998) 105, hep-th/9802109.

[3] E. Witten, "Anti-de Sitter space and holography," Adv. Theor. Math. Phys. 2 (1998) 253, hep-th/9802150.

[4] O. Aharony, S. S. Gubser, J. Maldacena, H. Ooguri, and Y. Oz, "Large N field theories, string theory and gravity," hep-th/9905111.

[5] L. Randall and R. Sundrum, "An alternative to compactification," hep-th/9906064.

[6] H. Verlinde, "Holography and compactification," hep-th/9906182.

[7] I. R. Klebanov, "World-volume approach to absorption by non-dilatonic branes," Nucl. Phys. B496 (1997) 231, hep-th/9702076.

[8] S. S. Gubser, I. R. Klebanov, and A. A. Tseytlin, "String theory and classical absorption by three-branes," Nucl. Phys. B499 (1997) 217, hep-th/9703040.

[9] S. S. Gubser and I. R. Klebanov, "Absorption by branes and Schwinger terms in the world volume theory," Phys. Lett. B413 (1997) 41-48, hep-th/9708005.

[10] J. Maldacena, private communications.

[11] H. Verlinde, invited talk at ITP Santa Barbara conference, "New Dimensions in Field Theory and String Theory," http://www.itp.ucsb.edu/online/susy_c99/verlinde/. 
[12] E. Witten, remarks at ITP Santa Barbara conference, "New Dimensions in Field Theory and String Theory," http://www.itp.ucsb.edu/online/susy_c99/discussion/.

[13] R. Sundrum, invited talk at ITP Santa Barbara conference, "New Dimensions in Field Theory and String Theory," http://www.itp.ucsb.edu/online/susy_c99/sundrum/.

[14] S. Giddings, invited talk at ITP Santa Barbara conference, "New Dimensions in Field Theory and String Theory," http://www.itp.ucsb.edu/online/susy_c99/giddings/.

[15] J. Polchinski and E. Witten, "Evidence for Heterotic - Type I String Duality," Nucl. Phys. B460 (1996) 525-540, hep-th/9510169.

[16] P. Horava and E. Witten, "Heterotic and Type I String Dynamics from Eleven Dimensions," Nucl. Phys. B460 (1996) 506-524, hep-th/9510209.

[17] M. Cvetic, S. Griffies, and H. H. Soleng, "Local and global gravitational aspects of domain wall space-times," Phys. Rev. D48 (1993) 2613-2634, gr-qc/9306005.

[18] A. Lukas, B. A. Ovrut, and D. Waldram, "Cosmological solutions of Horava-Witten theory," Phys. Rev. D60 (1999) 086001, hep-th/9806022.

[19] H. A. Chamblin and H. S. Reall, "Dynamic dilatonic domain walls," Nucl. Phys. B562 (1999) 133, hep-th/9903225.

[20] A. Chamblin, M. J. Perry, and H. S. Reall, "Non-BPS D8-branes and dynamic domain walls in massive IIA supergravities," JHEP 09 (1999) 014, hep-th/9908047.

[21] P. Kraus, "Dynamics of anti-de Sitter domain walls," hep-th/9910149.

[22] S. Mukohyama, "Brane-world solutions, standard cosmology, and dark radiation," hep-th/9911165.

[23] P. Binetruy, C. Deffayet, U. Ellwanger, and D. Langlois, "Brane cosmological evolution in a bulk with cosmological constant," hep-th/9910219.

[24] A. Kehagias and E. Kiritsis, "Mirage cosmology," JHEP 11 (1999) 022, hep-th/9910174.

[25] T. Shiromizu, K. Maeda, and M. Sasaki, "The Einstein equation on the 3-brane world," gr-qc/9910076. 
[26] E. E. Flanagan, S.-H. H. Tye, and I. Wasserman, "Cosmological expansion in the Randall-Sundrum brane world scenario," hep-ph/9910498.

[27] T. Nihei, "Inflation in the five-dimensional universe with an orbifold extra dimension," Phys. Lett. B465 (1999) 81-85, hep-ph/9905487.

[28] N. Kaloper, "Bent domain walls as braneworlds," Phys. Rev. D60 (1999) 123506, hep-th/9905210.

[29] O. DeWolfe, D. Z. Freedman, S. S. Gubser, and A. Karch, "Modeling the fifth dimension with scalars and gravity," hep-th/9909134.

[30] C. Csaki, M. Graesser, L. Randall, and J. Terning, "Cosmology of Brane Models with Radion Stabilization," hep-ph/9911406.

[31] D. N. Vollick, "Cosmology on a Three-Brane," hep-th/9911181.

[32] R. Brustein and G. Veneziano, "The Graceful exit problem in string cosmology," Phys. Lett. B329 (1994) 429-434, hep-th/9403060.

[33] M. Henningson and K. Skenderis, "The holographic Weyl anomaly," JHEP 07 (1998) 023, hep-th/9806087.

[34] B. A. Ovrut, "N=1 supersymmetric vacua in heterotic M-theory," hep-th/9905115.

[35] E. Witten and S. T. Yau, "Connectedness of the boundary in the AdS/CFT correspondence," hep-th/9910245.

[36] S. S. Gubser, I. R. Klebanov, and A. W. Peet, "Entropy and Temperature of Black 3-Branes," Phys. Rev. D54 (1996) 3915-3919, hep-th/9602135.

[37] A. Strominger, unpublished.

[38] V. Balasubramanian and P. Kraus, "A stress tensor for anti-de Sitter gravity," hep-th/9902121.

[39] L. Randall and R. Sundrum, "A large mass hierarchy from a small extra dimension," Phys. Rev. Lett. 83 (1999) 3370-3373, hep-ph/9905221.

[40] L. Susskind and E. Witten, "The holographic bound in anti-de Sitter space," hep-th/9805114.

[41] A. W. Peet and J. Polchinski, "UV/IR relations in AdS dynamics," Phys. Rev. D59 (1999) 065011, hep-th/9809022. 
[42] C. Fefferman and C. R. Graham, "Conformal invariants." in Elie Cartan et les Mathématiques d'aujourd'hui (Astérisque, 1985) 95.

[43] N. Arkani-Hamed, S. Dimopoulos, and G. Dvali, "The hierarchy problem and new dimensions at a millimeter," Phys. Lett. B429 (1998) 263, hep-ph/9803315.

[44] H. Liu and A. A. Tseytlin, " $\mathrm{D}=4$ super Yang-Mills, $\mathrm{D}=5$ gauged supergravity, and D $=4$ conformal supergravity," Nucl. Phys. B533 (1998) 88, hep-th/9804083.

[45] S. Giddings, E. Katz, and Lisa Randall, to appear.

[46] N. Arkani-Hamed, S. Dimopoulos, and G. Dvali, "Phenomenology, astrophysics and cosmology of theories with sub-millimeter dimensions and $\mathrm{TeV}$ scale quantum gravity," Phys. Rev. D59 (1999) 086004, hep-ph/9807344.

[47] I. Antoniadis, N. Arkani-Hamed, S. Dimopoulos, and G. Dvali, "New dimensions at a millimeter to a Fermi and superstrings at a TeV," Phys. Lett. B436 (1998) 257 , hep-ph/9804398.

[48] A. M. Polyakov, "The wall of the cave," Int. J. Mod. Phys. A14 (1999) 645, hep-th/9809057.

[49] S. Weinberg and E. Witten, "Limits on massless particles," Phys. Lett. B96 (1980) 59.

[50] E. Witten, "Anti-de Sitter space, thermal phase transition, and confinement in gauge theories," Adv. Theor. Math. Phys. 2 (1998) 505, hep-th/9803131.

[51] P. H. Frampton and C. Vafa, "Conformal approach to particle phenomenology," hep-th/9903226.

[52] L. Girardello, M. Petrini, M. Porrati, and A. Zaffaroni, "Novel local CFT and exact results on perturbations of $\mathrm{N}=4$ super Yang-Mills from AdS dynamics," JHEP 12 (1998) 022, hep-th/9810126.

[53] J. Distler and F. Zamora, "Non-supersymmetric conformal field theories from stable anti-de Sitter spaces," Adv. Theor. Math. Phys. 2 (1999) 1405, hep-th/9810206.

[54] S. S. Gubser, "Dilaton-driven confinement," hep-th/9902155.

[55] A. Kehagias and K. Sfetsos, "On running couplings in gauge theories from type-IIB supergravity," Phys. Lett. B454 (1999) 270, hep-th/9902125. 
[56] D. Z. Freedman, S. S. Gubser, K. Pilch, and N. P. Warner, "Renormalization group flows from holography supersymmetry and a c-theorem," hep-th/9904017.

[57] L. Girardello, M. Petrini, M. Porrati, and A. Zaffaroni, "The supergravity dual of $\mathrm{N}=1$ superYang-Mills theory," hep-th/9909047.

[58] J. Lykken and L. Randall, "The Shape of gravity," hep-th/9908076. 